\title{
CORRIGENDUM \\ Dengue infections in non-immune travellers to Thailand - CORRIGENDUM
}

\section{E. MASSAD, J. ROCKLOV AND A. WILDER-SMITH}

doi:10.1017/S0950268812000507, Published by Cambridge University Press, 24 April 2012.

An incorrect value for Mosquitoes Natural Mortality rate was given in Table 1 of the paper by E. Massad, J. Rocklov and A. Wilder-Smith. [1] Table 1 is republished here with the correct value.

Table 1. Model's parameters, biological meaning, values and sources

\begin{tabular}{|c|c|c|c|}
\hline Parameter & Meaning & Value & Source \\
\hline$a$ & Average Daily Biting rate & $0 \cdot 164$ & {$[15]$} \\
\hline$b$ & Fraction of actually infective bites & $0 \cdot 088$ & Fitted to data \\
\hline$\mu_{\mathrm{H}}$ & Humans Natural Mortality rate & $3 \cdot 5 \times 10^{-5}$ days $^{-1}$ & {$[16]$} \\
\hline$r_{\mathrm{H}}$ & Birth rate of humans & 8 days -1 & {$[16]$} \\
\hline$\kappa_{\mathrm{H}}$ & Humans Carrying Capacity & $16 \times 10^{6}$ & {$[16]$} \\
\hline$\alpha_{\mathrm{H}}$ & Dengue Mortality in Humans & $10^{-3}$ days $^{-1}$ & {$[17]$} \\
\hline$\gamma_{\mathrm{H}}$ & Humans recovery rate & $0 \cdot 143$ days $^{-1}$ & {$[17]$} \\
\hline$p_{\mathrm{S}}$ & Susceptible eggs hatching rate & $0 \cdot 15$ days -1 & {$[18]$} \\
\hline$d_{1}$ & Winter modulation parameter & $0 \cdot 07$ & assumed \\
\hline$d_{2}$ & Winter modulation parameter & $0 \cdot 06$ & assumed \\
\hline$\gamma_{\mathrm{M}}$ & Mosquitoes Latency rate & $0 \cdot 143$ days $^{-1}$ & \\
\hline f & Frequency of seasonal cycles & $2 \cdot 8 \times 10^{-3}$ days $^{-1}$ & assumed \\
\hline$\mu_{\mathrm{M}}$ & Mosquitoes Natural Mortality rate & $0 \cdot 09$ days -1 & [19] \\
\hline$\alpha_{\mathrm{M}}$ & Dengue Mortality in Mosquitoes & Negligible & - \\
\hline$r_{\mathrm{M}}$ & Oviposition rate & 50 days $^{-1}$ & [19] \\
\hline$p_{\mathrm{I}}$ & Infected eggs hatching rate & $0 \cdot 15$ days $^{-1}$ & [19] \\
\hline$g$ & Proportion of infected eggs & $0 \cdot 5$ & assumed \\
\hline$\kappa_{\mathrm{E}}$ & Eggs Carrying Capacity & $9 \cdot 8 \times 10^{7}$ & assumed \\
\hline$\mu_{\mathrm{E}}$ & Eggs Natural Mortality rate & $0 \cdot 1$ days $^{-1}$ & {$[19]$} \\
\hline$c$ & A.aegypti susceptibility to dengue & $0 \cdot 087$ & Fitted to data \\
\hline
\end{tabular}

\section{REFERENCE}

1. Massad E, Rocklov J and Wilder-Smith A. Dengue infections in non-immune travellers to Thailand. Epidemiology and Infection. Published by Cambridge University Press, 24 April 2012. doi:10.1017/S0950268812000507. 